\title{
Comprehensive description of the electro-optic effects in strained silicon waveguides
}

\author{
Pedro Damas, ${ }^{1}$ Mathias Berciano, ${ }^{1,}$ a) Guillaume Marcaud,${ }^{1}$ Carlos Alonso Ramos, ${ }^{1}$ Delphine Marris-Morini, ${ }^{1}$ Eric \\ Cassan, ${ }^{1}$ and Laurent Vivien ${ }^{1, b)}$ \\ Centre for Nanoscience and Nanotechnology (C2N), Université Paris Sud, CNRS, UMR 9001, \\ Université Paris-Saclay, Bât. 220, 91405 Orsay Cedex, France
}

We present a novel and comprehensive analysis method that considers both plasma-dispersion effect and the strain-induced Pockels effect to faithfully describe the electro-optic effects taking place in a strained silicon waveguide under an applied voltage. The change of carrier distribution arising from the application of a voltage, leads to a redistribution of the electrostatic field which deeply affects the strain-induced Pockels effect. By simulating the strain gradients distribution inside the waveguide together with the free carrier concentration in silicon, we were able to describe that the effective index change due to Pockels effect in strained silicon waveguides and the applied voltage have a nonlinear relationship.

\section{INTRODUCTION}

In recent years, silicon-based photonics has received much attention for its promising application in communications and optical interconnects developed in a CMOS platform $^{1}$. The major driver for silicon photonics is the possible development of electronics and photonics circuits in the same platform at reduced cost, while reducing the power consumption and increasing the speed of data transmission ${ }^{2}$.

The Pockels effect, an ultra fast electro-optic (E-O) effect (with temporal response as fast as femtosecond ${ }^{3}$ ) is commonly used in high speed and low-power optical modulators. But because of the inversion symmetry present in its crystal structure, silicon cannot exhibit any second order nonlinear effect such as Pockels effect. For that reason, optical modulation in silicon photonics is usually achieved using the free-carriers plasma dispersion effect ${ }^{17}$. However modulators based on this effect demonstrate high power consumption $(\sim 1 \mathrm{pJ} / \mathrm{bit})$ and their speed is limited by carriers mobility and recombination time.

One possible way to allow the realization of high performance Pockels-based modulators in silicon is to locally break its crystal symmetry by applying strain (typically through the use of a stress-overlayer made of SiN). This has been suggested and demonstrated in previous works on strained-silicon waveguides for Pockels effect ${ }^{4-8}$ and Second Harmonic Generation (SHG) ${ }^{9,10}$.

However, recently, it has been also demonstrated that free carriers inside silicon strongly contribute to the E-O effects in the strained silicon waveguides and the reason for the detected linear behavior between applied voltage and change in refractive index was the existence of charged centers in the SiN stress-layer ${ }^{11-13}$. This demonstration not only showed that the previous experimental results that did not take into account any free-carrier effects had been erroneously interpreted, but also that the

\footnotetext{
a) Electronic mail: mathias.berciano@c2n.upsaclay.fr

b) Electronic mail: laurent.vivien@c2n.upsaclay.fr
}

description of the E-O effects in strained silicon was being drastically over simplified. In fact, previous studies that considered silicon to be a regular dielectric whose freecarriers did not play a role led oversimplified descriptions of the strain induced E-O effect, giving rise to incomplete designing methodologies ${ }^{14}$ and experimental over estimations of $\chi^{(2) 6,7}$.

In this paper we present a description of the E-O effects occurring in strained silicon waveguides including both Pockels and plasma dispersion effects. We put together the strain, optical and carrier effects in a single model for its complete analysis. First, we start by describing the physics taking place when a strained silicon waveguide is subjected to a voltage change and how it affects both its refractive index and electric-field. Then, we proceed to the quantitative analysis of all the possible E-O effects, using recent advancements in the theoretical description of strain-induced $\chi^{(2)}$ using strain gradients and merging it with the carrier distribution in the waveguide under a voltage. Finally, we put all the effects together to give estimations and trends of the overall E-O response of a strained silicon waveguide as a function of an applied voltage.

\section{THE PHYSICAL ORIGINS OF THE ELECTRO-OPTIC EFFECTS}

There are different E-O effects that can take place inside a strained silicon waveguide and contribute for a variation of the dielectric permittivity $(\Delta \epsilon)$ of silicon, when an electrostatic field $(\boldsymbol{F})$ is present in the waveguide. Those include the plasma-dispersion effect $\left(\Delta n_{\text {eff }}^{c}\right.$, $c$ standing for carriers), the Kerr effect $\left(\Delta n_{\text {eff }}^{K}\right)$ and the Pockels effect $\left(\Delta n_{\text {eff }}^{P}\right)$, this one being possible only in strained waveguides, where the centro-symmetry is broken. The final E-O effect is thus given by the sum of all the mentioned contributions, so that

$$
\Delta n_{\mathrm{eff}}=\Delta n_{\mathrm{eff}}^{c}+\Delta n_{\mathrm{eff}}^{P}+\Delta n_{\mathrm{eff}}^{K} .
$$

For our analysis on the E-O effects in a strained silicon waveguide, we concentrated on the structure typi- 


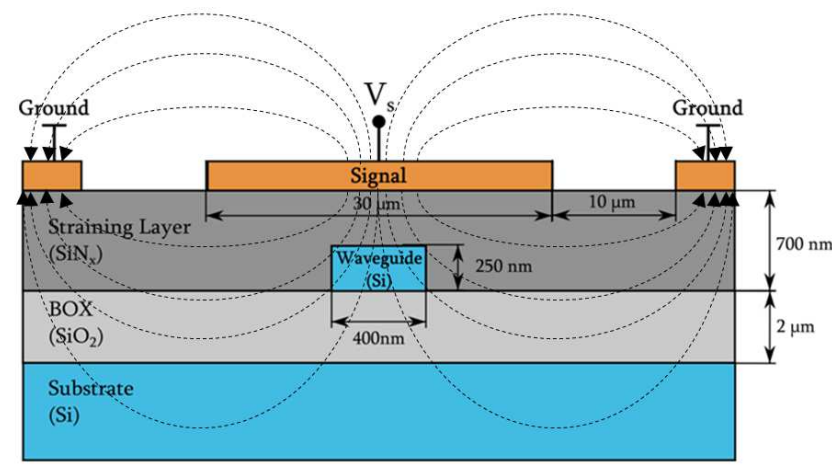

FIG. 1: Cross-section of the strained waveguide structure. The stress SiN layer (internal stress $\left.\sigma_{0}=1 \mathrm{GPa}\right)$ strains the waveguide underneath. The electrodes generate an electric field $(\boldsymbol{F})$ in the waveguide.

cally used in strained silicon for Pockels modulation ${ }^{5-7}$, depicted in Fig. 1: a p-doped silicon waveguide (acceptor density $N_{A} \sim 1 \times 10^{15} \mathrm{~cm}^{-3}$ ), 250nm-high and 400nmwide, is covered by a $700 \mathrm{~nm}$-thick stressed SiN overlayer (with internal stress of $1 \mathrm{GPa}$ ), which strains the waveguide underneath, breaking its centro-symmetry.

The concentration of electrons and holes, $n$ and $p$, respectively, inside the strained silicon waveguide may change due to a variation of the electrical potential $(V(\boldsymbol{r}))$ in the structure. The physical process that leads to this variation of carriers concentration has the same physical roots as that occurring in the widely studied Metal-Oxide-Semiconductor (MOS) devices and can be explained as follows ${ }^{15}$ : when a voltage $V_{s} \neq 0$ is applied to the signal electrode in Fig. 1, $V(\boldsymbol{r})$ is changed inside the structure, leading to a shift of the Fermi level $\left(E_{F}\right)$ and to the bending of the energy bands (valence and conduction) in silicon. The final free-carrier concentration is the result of the interplay between the band bending and the $E_{F}$ shift induced by the applied voltage $V_{s}$. An extensive description of these processes can be found elsewhere ${ }^{15}$.

In order to study the described phenomena, we developed a simulation model of the structure depicted in Fig. 1. The simulations have been performed using three modules, namely:

- A modal analysis module to compute the optical TE mode propagating through the silicon waveguide.

- A solid mechanics module to compute the strain gradient fields induced by the silicon nitride stressed overlayer on the silicon waveguide.

- A semiconductor module solving the Poisson's equation, the drift-diffusion equations and the continuity equations to compute the concentration of carriers inside the waveguide and the electrostatic field $\boldsymbol{F}$.

For each modules used, a finite element method has been employed to perform the calculations. In the simula- tions, we varied the voltage applied to the signal electrode $\left(V_{s}\right)$ and explored how it affects the free-carrier distribution inside the waveguide. Fig. 2 reports on the total number of electrons and holes inside the waveguide as a function of $V_{s}$, together with the corresponding crosssectional plots of the holes (majority carriers) concentrations for three different voltages levels: $V_{s}=-10 \mathrm{~V}, 2 \mathrm{~V}$ and $10 \mathrm{~V}$. These three voltages correspond respectively, to some degree, to three well known regimes in MOS devices: accumulation, where the majority carrier concentration dominates (the conduction band is very close to $\left.E_{F}\right)$; depletion, where the concentration of any of the free-carriers is very low ( $E_{F}$ is far from both the conduction and valence bands) and centered at $V_{s}=V_{D}$; and inversion, where the minority carrier concentration (electrons) overcomes that of the majority carriers $\left(E_{F}\right.$ is closer to the valence band). It must be emphasised that the depletion voltage is different from the flatband for which both conduction and valence bands are flat nearby the waveguide interfaces, leading to a higher concentration of holes than electrons in the waveguide.

The previous simulations did not take into account any charging process of the cladding. In fact, it has been reported $^{11-13}$ that the SiN cladding is responsible for the creation of a positive surface fixed charge density $Q_{f}$ at the semiconductor-SiN interface. Indeed during the deposition of This charge density results in an effective voltage shift $\Delta V$ in the $p\left(V_{s}\right)$ and $n\left(V_{s}\right)$ behaviours. This is shown in Fig 2, obtained from simulations where we included $Q_{f}=3 \times 10^{12} \mathrm{~cm}^{-2}$ at the Si/SiN interface, as measured by Sharma et al. in ${ }^{12}$. Due to the MaxwellBoltzmann statistics approach used in the simulations, the total number of carriers in the waveguide increases indefinitely because of the bending of the energy bands (the number of carriers depends exponentially on the energy level value). Those fixed charges then modified both free-carriers plasma dispersion effect and Pockels effect. In the following, we performed a comparison between the uncharged $\left(Q_{f}=0\right)$ and charged $\left(Q_{f} \sim 3 \times 10^{12} \mathrm{~cm}^{-2}\right)$ states.

The carrier concentration has direct influence on the refractive index of the structure through the plasmadispersion effect ${ }^{16,17}$. However, it also indirectly affects the Pockels and Kerr effects because the electrostatic field distribution $(\boldsymbol{F})$ inside the waveguide is dependent on the carrier distribution. In Fig. 3 we present the crosssectional contour plot of $F_{y}$ together with the vectorial field of $\boldsymbol{F}$ in the waveguide region for the three regimes described above. We clearly see that it is highly inhomogeneous in the waveguide and extremely dependent on the applied voltage. The free-carrier distribution has a strong influence on the distribution of the electrostatic field inside the waveguide, which is in the origin of the electro-optic effects.

As a result, the application of a voltage $V_{s}$ in the system modifies the free-carrier density in the silicon waveguide (affecting the $\Delta n_{\text {eff }}^{c}$ ), which in turn changes the elestrostatic field distribution in the waveguide (affecting 

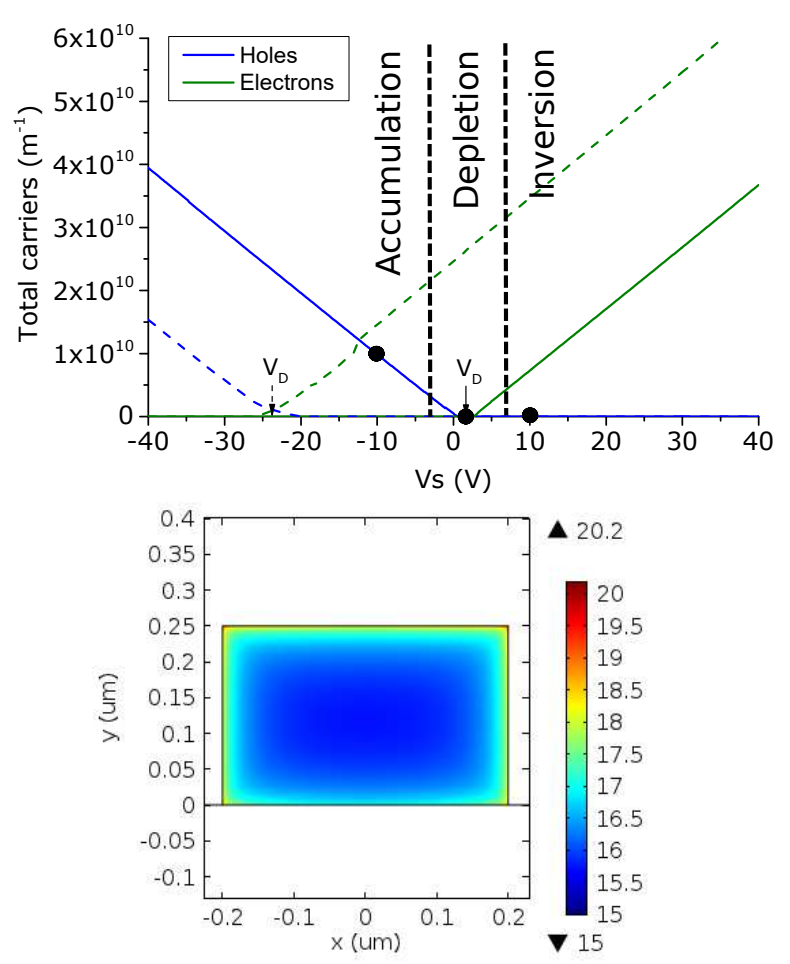

(a) $V_{s}=-10 \mathrm{~V}$

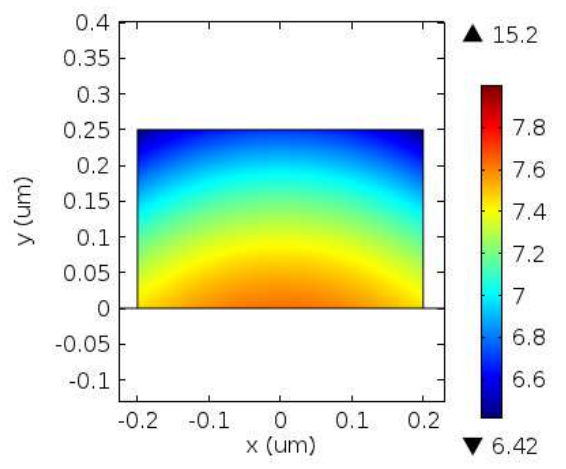

(b) $V_{s}=2 \mathrm{~V}$

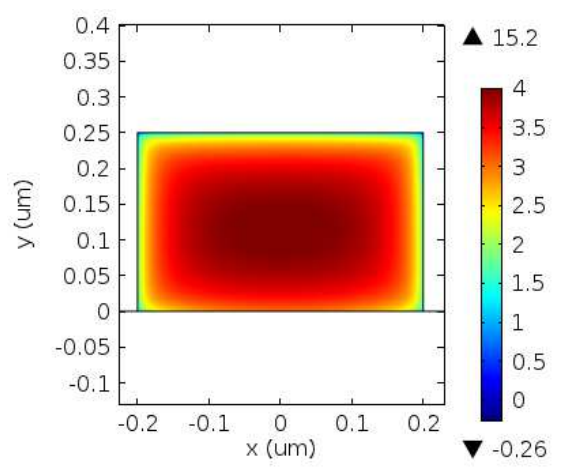

(c) $V_{s}=10 \mathrm{~V}$

FIG. 2: The total number of carriers in the waveguide as a function of the applied voltage for uncharged $\left(Q_{f}=0\right.$, solid $)$ and charged $\left(Q_{f} \sim 3 \times 10^{12} \mathrm{~cm}^{-2}\right.$, dashed) cases of the cladding, with the identification of the three regimes and depletion voltage $V_{D}$. The corresponding cross-section distribution (in logarithmic scale) of the hole concentration $p\left(\right.$ in $\left.\mathrm{cm}^{-3}\right)$ for the three different regimes a), b) and c).

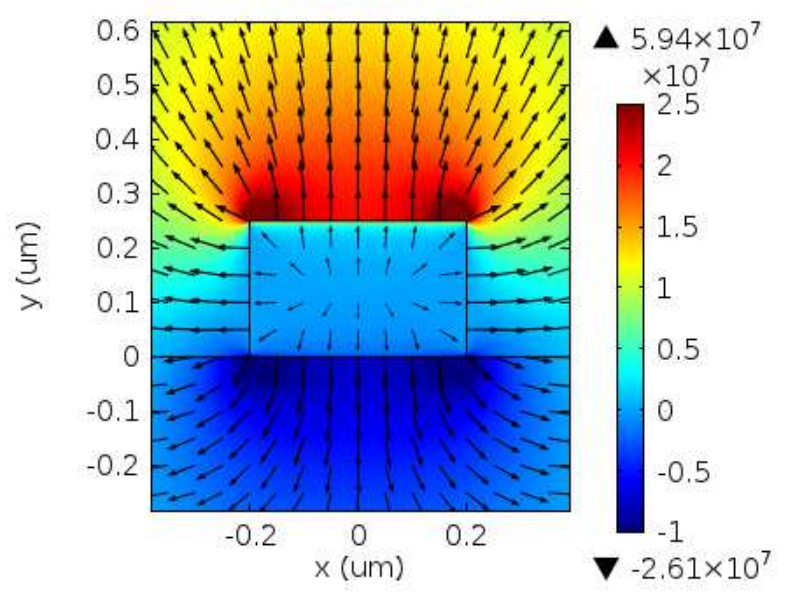

(a) $V_{s}=-10 \mathrm{~V}$

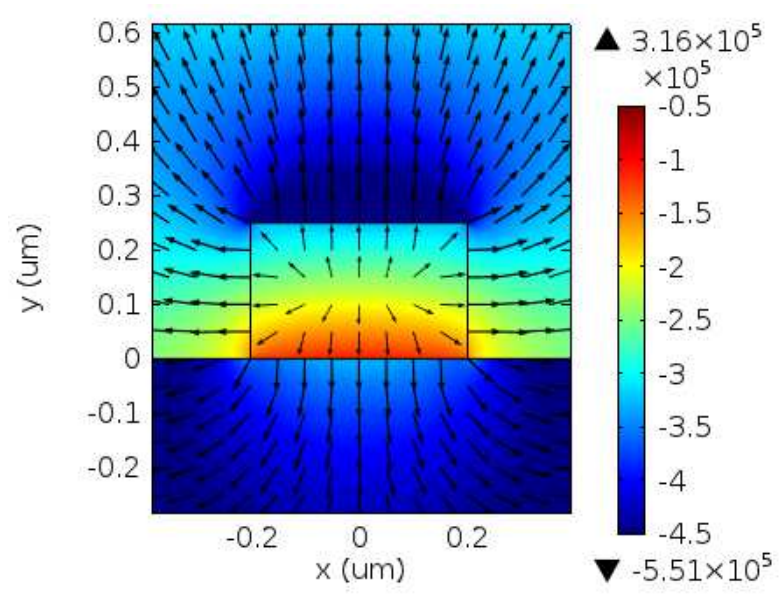

(b) $V_{s}=2 \mathrm{~V}$

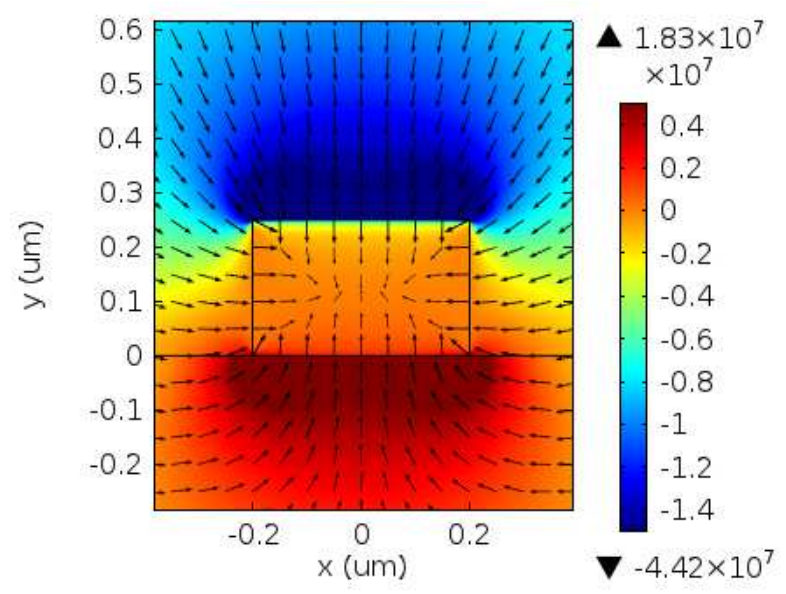

(c) $V_{s}=10 \mathrm{~V}$

FIG. 3: The distribution of the $F_{y}$ field (color), together with the vector field $\boldsymbol{F}$ (arrow surface) in the uncharged waveguide $\left(Q_{f}=0\right)$ in the region of the waveguide, for the three different regimes: a) accumulation, b) depletion and c) inversion. 
both $\Delta n_{\mathrm{eff}}^{P}$ and $\left.\Delta n_{\mathrm{eff}}^{K}\right)$. This redistribution is highly nonlinear inside the waveguide, as it can be seen in Fig. 3 and results in a nonlinear relation between $F_{y}$ and $V_{s}$. This nonlinear phenomenon is the main reason for the differences between the E-O description including the freecarrier effects and the simplified analysis considering the waveguide as a dielectric material ${ }^{5-7,14}$.

\section{QUANTITATIVE DESCRIPTION OF THE ELECTRO-OPTIC EFFECTS}

In every electro-optic effect the dielectric permittivity tensor $(\overline{\boldsymbol{\epsilon}})$ of a material undergoes a variation

$$
\overline{\boldsymbol{\epsilon}}=\overline{\boldsymbol{\epsilon}}^{0}+\Delta \overline{\boldsymbol{\epsilon}},
$$

where $\overline{\boldsymbol{\epsilon}}^{0}$ is the original permittivity tensor of the material and $\Delta \overline{\boldsymbol{\epsilon}}$ is the E-O effect induced by the applied electric field and generally we may assume that $\Delta \overline{\boldsymbol{\epsilon}} \ll \overline{\boldsymbol{\epsilon}}^{0}$.

The change in dielectric permittivity of the material induces a variation of the effective index $n_{\text {eff }}$ of the optical mode propagating in the waveguide. If the electric and magnetic profiles of the mode propagating in the $z$ direction are given by $\boldsymbol{E}(x, y)$ and $\boldsymbol{H}(x, y)$, respectively, it can be shown that $\Delta n_{\text {eff }}$ can be generally given by ${ }^{14}$ :

$$
\Delta n_{\mathrm{eff}}=\frac{1}{N} \int \boldsymbol{E} \cdot \Delta \overline{\boldsymbol{\epsilon}} \cdot \boldsymbol{E}^{*} d x d y
$$

where the integration is carried over the entire space. The parameter $N$ corresponds to the active power of the mode and it is given by

$$
N=\frac{1}{c \epsilon_{0}} \int\left(\boldsymbol{E} \times \boldsymbol{H}^{*}+\boldsymbol{E}^{*} \times \boldsymbol{H}\right) \cdot \hat{\boldsymbol{z}} d S .
$$

Equation 3 allows calculating $\Delta n_{\text {eff }}$ for given mode and $\Delta \overline{\boldsymbol{\epsilon}}$ profiles. In this analysis we only concentrated on the TE-fundamental mode of the waveguide structure presented in Fig. 1. Thus, what is left to evaluate is the specific $\Delta \overline{\boldsymbol{\epsilon}}$ resulting from each one of the three E-O effects discussed in the previous section.

First, Kerr-effect, which is a quadratic E-O mediated by the third order nonlinear susceptibility tensor $\overline{\bar{\chi}}^{(3)}$ of the material, so that the change of dielectric permittivity $\overline{\boldsymbol{\epsilon}}_{K}(\boldsymbol{r})$ is given by

$$
\Delta \overline{\boldsymbol{\epsilon}}_{K}=3 \overline{\bar{\chi}}^{(3)}: \boldsymbol{F} \boldsymbol{F} .
$$

In silicon, $\chi^{(3)} \sim 1 \times 10^{-19} \mathrm{~m}^{2} / \mathrm{V}^{218}$ at $\lambda=1.55 \mu \mathrm{m}$ and from Fig 3, the electric field inside the waveguide takes values $F_{y} \sim 1 \times 10^{6} \mathrm{~V} / \mathrm{m}$. Inserting these values in eq. 3 leads to $\Delta n_{\text {eff }}^{K}$ from $1 \times 10^{-7}$ to $1 \times 10^{-6}$, in the voltage range we considered. This confirms the widely known statement that Kerr effect is very weak in silicon and plays a negligible role in the E-O effects, even considering the fixed charges at the interface of the waveguide. Consequently, it may be neglected when compared with stronger contributions like plasma-dispersion and straininduced Pockels effects, which is the focus of the following analysis.

\section{A. Plasma-Dispersion effect}

The change in refractive index of silicon due to the change in free-carrier concentration (plasma-dispersion effect) is given by the so-called Soref-Bennet equations which, at $\lambda=1.55 \mu \mathrm{m}$ are given $\mathrm{by}^{17}$ :

$$
\Delta n_{c}=-\left(8.8 \times 10^{-22} \Delta n+8.5 \times 10^{-18} \Delta p^{0.8}\right) .
$$

where $\Delta n$ and $\Delta p$ are respectively the electron and hole concentration change expressed in $\mathrm{cm}^{-3}$.

The corresponding change in permittivity is then given by

$$
\Delta \epsilon_{c}=2 n_{\mathrm{Si}} \Delta n_{c}
$$

which can be inserted in eq. 3 to give

$$
\Delta n_{\mathrm{eff}}^{c}=\frac{2 n_{\mathrm{Si}}}{N} \int_{w g} \boldsymbol{E}^{2} \cdot \Delta n_{c} d S
$$

The value of $\Delta n_{\mathrm{eff}}^{c}$ as a function of $V_{s}$ was calculated using the simulations described in the previous section together with eq. 6. The results, for both uncharged and charged cases are shown in Fig. 4. The curve is negative because of the minus sign in eq. 6 and the maximum voltage, occurring at $V_{s}=V_{D}$, corresponds to the depletion regime of the system, where both electrons and holes concentrations are low. Away from this point, the $\Delta n_{\text {eff }}^{c}$ decreases because of the increasing number of free-carriers in both accumulation and inversion regimes. The position of the depletion regime is, as expected, dependent on $Q_{f}$, corresponding to $V_{D} \sim 2 \mathrm{~V}$ and $V_{D} \sim-24 \mathrm{~V}$ for the uncharged $\left(Q_{f}=0\right)$ and charged $\left(Q_{f}=3 \times 10^{12} \mathrm{~cm}^{-2}\right)$ situations, respectively. These results are in line with those reported in other publications on this subject ${ }^{11,12}$.

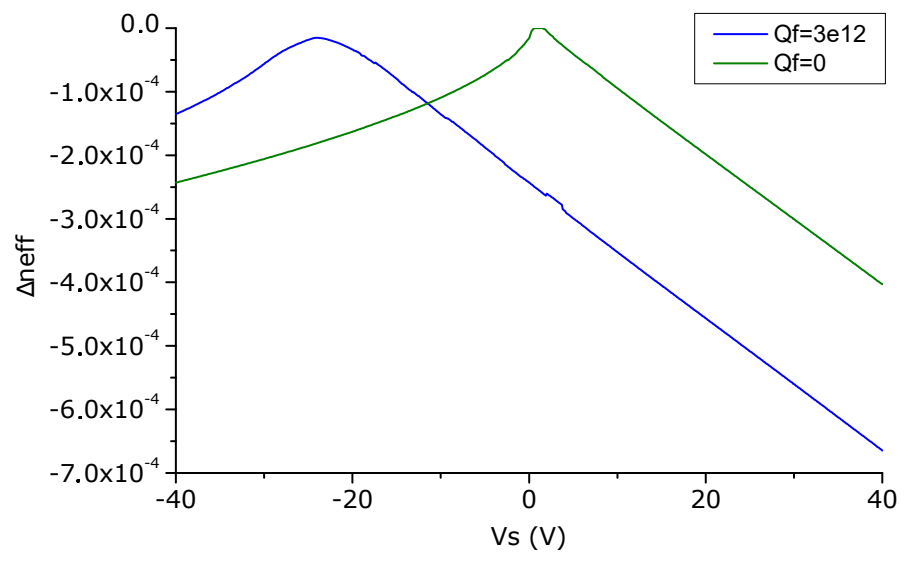

FIG. 4: The resulting $\Delta n_{\mathrm{eff}}^{c}$ as a function of the applied voltage $\left(V_{s}\right)$ for the structure represented in Fig. 1 for both uncharged $\left(Q_{f}=0\right.$, green) and charged $\left(Q_{f}=3 \times 10^{12} \mathrm{~cm}^{-2}\right.$, blue) situations. 


\section{B. Pockels-effect in strained silicon}

Pockels effect arises from the interaction between the electrostatic electric field $\boldsymbol{F}$ and the second order nonlinear susceptibility tensor $\overline{\bar{\chi}}^{(2)}$ of the material. The change of dielectric permittivity $\overline{\boldsymbol{\epsilon}}_{P}(\boldsymbol{r})$ is given by:

$$
\Delta \overline{\boldsymbol{\epsilon}}_{P}=2 \overline{\bar{\chi}}^{(2)} \cdot \boldsymbol{F}
$$

Inserting the previous equation into eq. 3, allows calculating the Pockels effective index change as:

$$
\Delta n_{\mathrm{eff}}^{P}=\frac{2}{N} \int_{w g} \chi_{i j k}^{(2)} E_{i} E_{j} F_{k} d S .
$$

The difficulty with the evaluation of the previous equation is in the value of $\chi^{(2)}$ to use, which must depend on the deformation of the crystal. It has been demonstrated that strain gradients are behind the generation of $\chi^{(2)}$ in centro-symmetric crystals ${ }^{14,19}$. By defining the strain gradient tensor $(\overline{\boldsymbol{\eta}})$ components by

$$
\eta_{i j k}=\frac{\partial \varepsilon_{i j}}{\partial x_{k}},
$$

$\chi^{(2)}$ can be generally written as ${ }^{14,19}$

$$
\chi_{i j k}^{(2)}=\Gamma_{i j k, l m n} \eta_{l m n} .
$$

The coefficients $\Gamma$ are dependent on the crystal properties. Despite the values of these parameters are not known, recently, we have demonstrated that these coefficients are dependent only on two parameters $\alpha$ and $\beta$, to be determined experimentally ${ }^{19}$.

Inserting eq. 12 into eq. 10, leads to the following relation:

$$
\Delta n_{\mathrm{eff}}^{P}=\Gamma_{i j k, m n l} \widehat{\eta_{m n l}^{i j k}}
$$

The quantities $\widehat{\eta_{m n l}^{i j k}}$ are named effective strain gradients and are defined by:

$$
\widehat{\eta_{m n l}^{i j k}}=\frac{2}{N} \int_{w g} \eta_{m n l} E_{i} E_{j} F_{k} d S,
$$

corresponding to the overlap integral between the optical mode $(E)$, strain gradients $(\eta)$ and electrostatic field $(F)$ spatial distributions inside the waveguide. The previous equation shows how Pockels effect depends on the interaction between the strain effects, optical mode and applied electrostatic field, which can be simulated independently.

In principle, all the components of the $\eta$ and $\chi^{(2)}$ tensors contribute to the Pockels effective index change, each contribution being weighted by the corresponding $\Gamma_{i j k, m n l}$ coefficient, which in this paper we consider to be unknown. However, the symmetries of the problem lead to a drastic simplification of the sum in eq. 14 because most of the terms vanish or are very small. For the TE-mode propagating in the structure of Fig. 1 the only relevant effective strain gradient components are $\widehat{\eta_{x x y} x y}$ and $\widehat{\eta_{y y y} x^{14}-19}$. Indeed while $F_{x}$ and $F_{y}$ components of the electrostatic field $\boldsymbol{F}$ are at equivalent magnitude, simulation results shown that $\widehat{\eta_{x x y}^{x x x}} \ll \widehat{\eta_{x x y} x y}$ and $\widehat{\eta_{y y y}^{x x x}} \ll \widehat{\eta_{y y y}^{x x y}}$.Therefore, $\Delta n_{\text {eff }}^{P}$ is reduced to the sum of only two terms, resulting in:

$$
\begin{aligned}
\Delta n_{\mathrm{eff}}^{P} & =\Gamma_{x x y, x x y} \widehat{\eta_{x x y}^{x x y}}+\Gamma_{x x y, y y y} \widehat{\eta_{y y y} y} \\
& =\Gamma_{x x y, x x y}\left(\widehat{\eta_{x x y}^{x x y}}+\zeta \widehat{\eta_{y y y} y}\right)
\end{aligned}
$$

where we have defined

$$
\zeta \equiv \frac{\Gamma_{x x y, y y y}}{\Gamma_{x x y, x x y}} .
$$

In the previous equation, $\Gamma_{x x y, x x y}$ contains the information about the intensity of the Pockels effect, affecting the numerical value of $\Delta n_{\mathrm{eff}}^{P}$, whereas the parameter $\zeta$ defines the change of the voltage dependent curve, because it sets the relative weight of $\widehat{\eta_{x x y}^{x x y}}$ and $\widehat{\eta_{y y y} y}$ effective gradients in the strain-induced Pockels effect.

The calculation of the strain gradients inside the waveguide was performed by simulating the deformation state of the structure when its strain is due to the SiN layer, with internal stress $\sigma_{0}=1 \mathrm{GPa}$. The crosssectional profiles of the strain gradient components $\eta_{x x y}$ and $\eta_{y y y}$ are shown in Fig. 5 .

The results for the $\eta_{x x y}$ and $\eta_{y y y}$ can be used together with the field profiles of Fig. 3 as a function of $V_{s}$ in order to calculate $\widehat{\eta_{x x y} x}\left(V_{s}\right)$ and $\widehat{\eta_{y y y}^{x x y}}\left(V_{s}\right)$. The result are presented in Fig. 6 and shows that $\widehat{\eta_{x x y}^{x x y}}$ and $\widehat{\eta_{y y y}^{x y}}$ have opposite signs, which comes from the difference in the sign of the distribution of the respective strain gradients, as shown in Fig. 5. Furthermore, it is clear that the effective strain gradients undergo a sign shift around $V_{D}$, where the sign of the electric field inside the waveguide change. In the region close to the $V_{D}$ (in the depletion regime), $\widehat{\eta}$ are very sensitive to voltage changes, stabilizing away from this point where the curve is considerably flat.

The final Pockels effective index change is a combination of the referred two effective gradients (see eq. 16) and the $\Gamma$ coefficients, which are unknown parameters that should be determined experimentally. Nonetheless, it order to provide information about the possible trends of the strain-induced Pockels effect, in Fig. 7 we present the $\Delta n_{\mathrm{eff}}^{P}\left(V_{s}\right)$ for different $\zeta$ values in the uncharged configuration. It clearly shows that the strain-induced Pockels effect curve shape enormously varied depending on the relative value of the $\Gamma$ coefficients.

\section{FINAL ELECTRO-OPTIC EFFECT}

The effects discussed in the previous sections can be now put together to calculate the final $\Delta n_{\text {eff }}$, as given in 


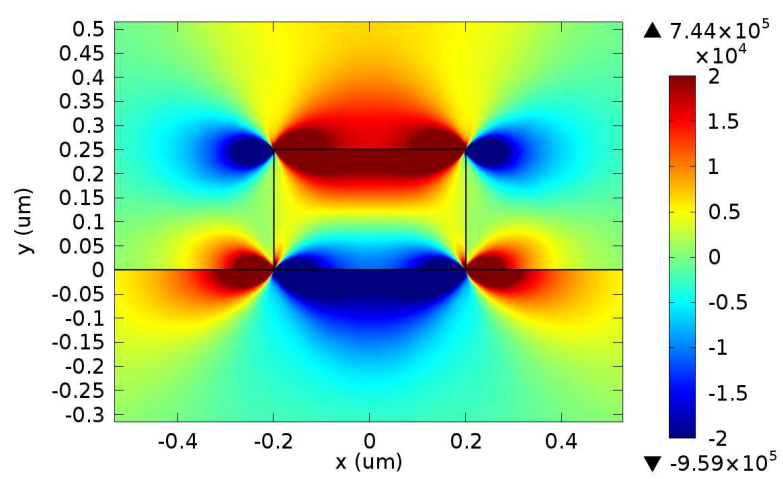

(a) $\eta_{x x y}$

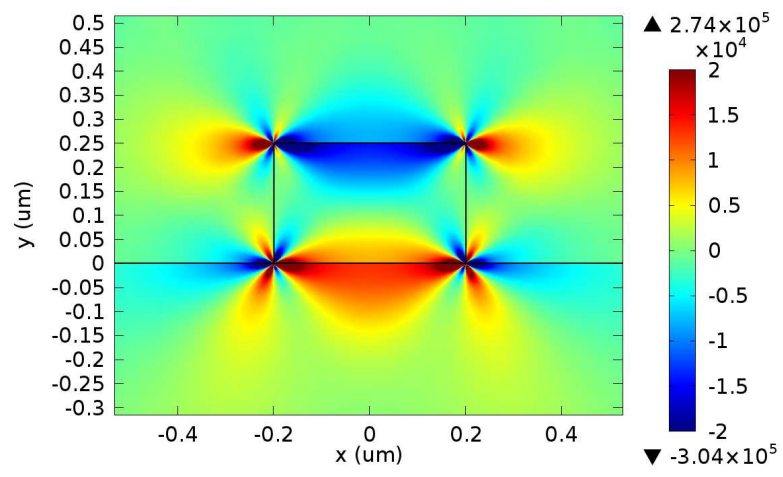

(b) $\eta_{y y y}$

FIG. 5: The distribution (in $\mathrm{m}^{-1}$ ) of the most relevant strain gradients components in the waveguide, represented by the rectangle in the center.

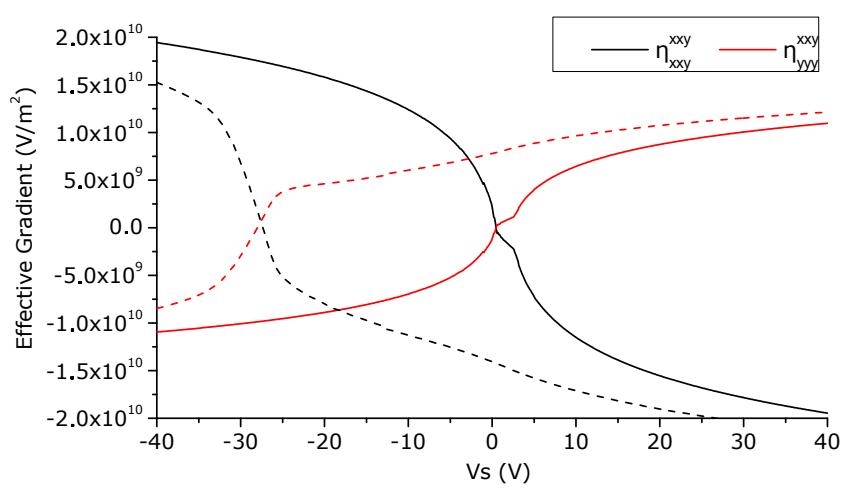

FIG. 6: The representation of the effective strain gradient components $\widehat{\eta_{x x y}^{x x y}}$ (black) and $\widehat{\eta_{y y y}^{x y}}$ (red) in the structure of Fig. 1 as a function of the applied voltage

$\left(V_{s}\right)$, for both uncharged $\left(Q_{f}=0\right.$, full $)$ and charged

$\left(Q_{f}=3 \times 10^{12} \mathrm{~cm}^{-2}\right.$, dashed) situations.

eq. 1. As discussed previously, we neglect the contribution from Kerr effect and the E-O effect is thus reduced

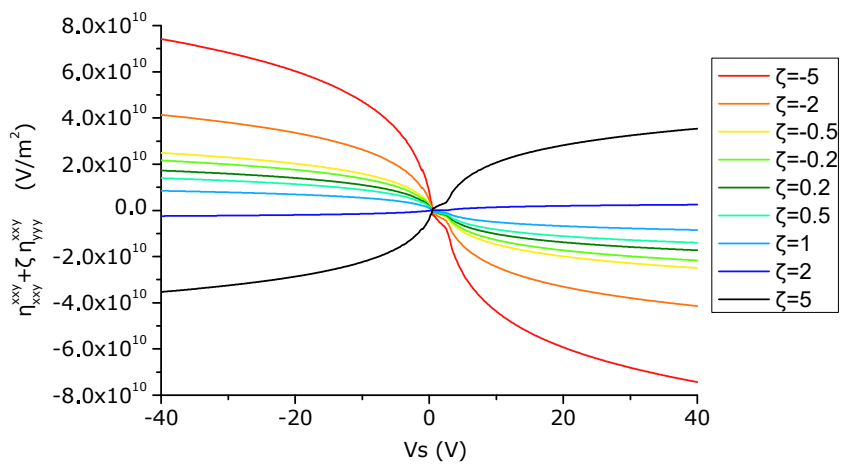

FIG. 7: The final value of $\widehat{\eta_{x x y}^{x x y}}+\zeta \widehat{\eta_{y y y}^{x y y}}$ for the uncharged case $\left(Q_{f}=0\right)$ of Fig. 6 for different values of $\zeta$ spanning from -5 to 5 .

to the plasma-dispersion and Pockels terms, leading to

$$
\begin{aligned}
\Delta n_{\mathrm{eff}} & =\Delta n_{\mathrm{eff}}^{c}+\Delta n_{\mathrm{eff}}^{P}= \\
& =\Delta n_{\mathrm{eff}}^{c}+\Gamma_{x x y, x x y}\left(\widehat{\eta_{x x y}^{x x y}}+\zeta \widehat{\eta_{y y y}^{x x y}}\right) .
\end{aligned}
$$

As mentioned before, without the knowledge of the $\Gamma$ coefficients, the analysis of the final electro-optic effect must be done as a function of both $\zeta$ and $\Gamma_{x x y, x x y}$ coefficients. The effects of a wide range of $\zeta$ values in the strain-induced $\chi^{(2)}$, is shown in Fig. 7 while for the analysis of the total E-O effect in the waveguide we only focused on two different values: $\zeta= \pm 1$. In this way, we explicitly calculate how two symmetric values of $\zeta$ affect the final E-O effect while keeping the analysis as simple as possible.

With $\zeta$ value fixed, the average $\chi^{(2)}$ inside the waveguide is given by

$$
<\chi_{x x y}^{(2)}>=\Gamma_{x x y, x x y}\left(<\eta_{x x y}> \pm<\eta_{y y y}>\right),
$$

where $<>$ stands for averaging inside the waveguide. From the previous equation, the value of $\left\langle\chi_{x x y}^{(2)}\right\rangle$ uniquely determines $\Gamma_{x x y, x x y}$ (and vice-versa), because the strain gradients average can be calculated from the simulations shown in Fig. 5 which give:

$$
\begin{array}{ll}
<\eta_{x x y}>+<\eta_{y y y}>=2737 \mathrm{~m}^{-1}, & \text { for } \zeta=1, \\
<\eta_{x x y}>-<\eta_{y y y}>=8922 \mathrm{~m}^{-1}, & \text { for } \zeta=-1 .
\end{array}
$$

Therefore, the rest of our analysis is carried out in terms of $\left\langle\chi_{x x y}^{(2)}\right\rangle$ because it provides a better understanding on the strength of the strain-induced Pockels effect and eases the comparison with previous experiments and materials. Therefore, in this analysis we considered $\left\langle\chi_{x x y}^{(2)}\right\rangle=1 \mathrm{pm} / \mathrm{V}, 10 \mathrm{pm} / \mathrm{V}$ and $50 \mathrm{pm} / \mathrm{V}$, values chosen based on previous publications. The first corresponds to the lowest values reported from Pockels effect in strained silicon ${ }^{20}$. The other two values, $10 \mathrm{pm} / \mathrm{V}$ and $50 \mathrm{pm} / \mathrm{V}$, correspond to higher values reported from previous works on the field ${ }^{4,9}$. 


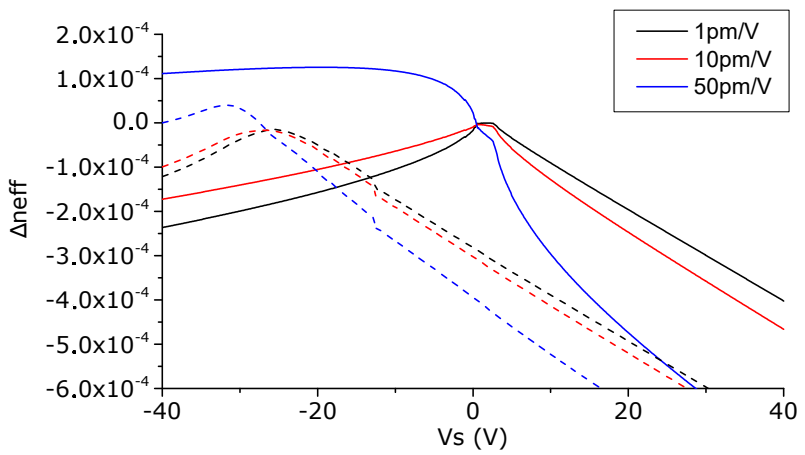

(a)

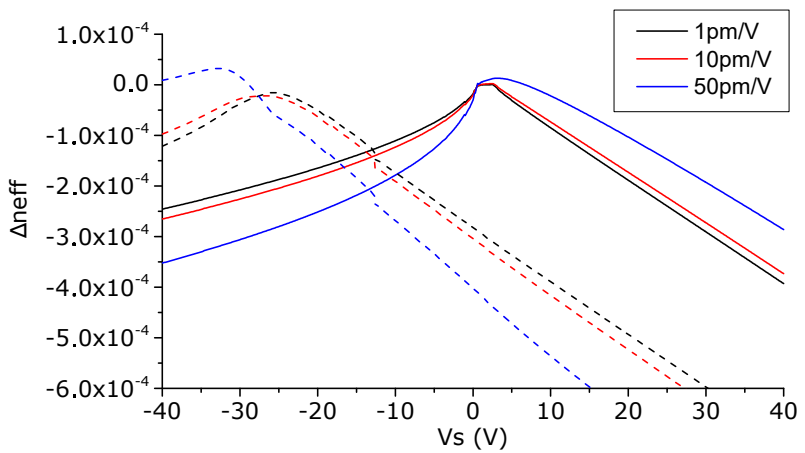

(b)

FIG. 8: The representation of the total electro-optic effect, constituted by the plasma-dispersion and strain-induced Pockels effect assuming levels of average $\chi^{(2)}$ inside the waveguide and considering (a) $\zeta=1$, (b) $\zeta=-1$. The solid line corresponds to the uncharged case, while the dashed line corresponds to the charged situation.

Figs. 8 shows the results for $\zeta=1$ and $\zeta=-1$, and for the uncharged (solid) and charged (dashed) situations. From the different changes undergone by the two curves, it is noticeable the effects of the increasing straininduced $\chi^{(2)}$ values on the overall E-O effect and their strong dependence on the $\zeta$ parameter. When considering $1 \mathrm{pm} / \mathrm{V}$, the $\Delta n_{\text {eff }}^{c}$ curve is not perturbed by the strain induced Pockels effect. Thus, if $\chi^{(2)}$ has indeed this order of magnitude, it is impossible to dissociate it from any DC experiments, as its effect is overtaken by the plasma-dispersion effect and $\Delta n_{\mathrm{eff}} \simeq \Delta n_{\mathrm{eff}}^{c}$.

However, if we consider the average $\chi^{(2)}$ in the waveguide to be $10 \mathrm{pm} / \mathrm{V}$, even though $\Delta n_{\text {eff }}^{c}$ is still the most relevant effect, we can detect a change in the curve behaviour which could possibly be detected experimentally. Finally, for $\left\langle\chi_{x x y}^{(2)}\right\rangle=50 \mathrm{pm} / \mathrm{V}$, both plasma-dispersion effect and the strain-induced $\chi^{(2)}$ have almost identical importance in the curve behaviour, leading to reasonable changes in the E-O curves presented in Figs. 8a and 8b. In this situation, we cannot consider $\Delta n_{\text {eff }}^{c}$ to be the dominant effect and only a joint analysis of both carriers and strain effects can lead to correct interpretations.

\section{CONCLUSION}

In this paper we thoroughly presented a comprehensive method that includes carrier effects to faithfully describe strain-induced Pockels effects in silicon waveguides. The underlying physical phenomena relating the carrier and Pockels effects arises from the carrier distribution modification in presence of an applied voltage, which in turn affects the electrostatic field in the strained silicon waveguide, thereby influencing the Pockels effect.

Under these considerations, and relying on the latest studies on the origin of Pockels effects in strained silicon, we developed a model which puts together the strain effects, the carriers and the optical modes together, to fully describe the electro-optic effect as a combination of both plasma-dispersion and Pockels effects. The final electrooptic effect depends on the strength of the strain-induced $\chi^{(2)}$. These estimated values of $\Delta n_{\text {eff }}$ have shown that only average $\chi^{(2)}$ values in the waveguide higher than $10 \mathrm{pm} / \mathrm{V}$ can have any relevance on the measured E-O effect.

The method proposed in this work, represent the backbone of any complete analysis of Pockels effects in strained-silicon waveguides, which must include the freecarriers in the waveguide and the charging effects of the cladding. We strongly believe that the analysis method we developed in this paper sets the basis for a deep understanding of strain-induced Pockels effect in silicon waveguide, opening a new route for the design and optimization of fast and low-power consumption Pockels-based silicon electro-optic modulators.

\section{ACKNOWLEDGMENTS}

Authors would like to thank Xavier Le Roux from C2N and Frédéric Boeuf from STMicroelectronics (Crolles, France) for fruitful discussions. The authors also acknowledge STMicroelectronics for the financial support of the P. Damas' scholarship. This project has received funding from the European Research Council (ERC) under the European Union's Horizon 2020 research and innovation program (ERC POPSTAR - grant agreement $\left.\mathrm{N}^{\mathrm{O}} 647342\right)$.

${ }^{1}$ H. Subbaraman, X. Xu, A. Hosseini, X. Zhang, Y. Zhang, D. Kwong, and R. T. Chen, Optics Express 23, 2487 (2015).

${ }^{2}$ D. Miller, "Device Requirements for Optical Interconnects to Silicon Chips," (2009).

${ }^{3}$ H. Lin, S. Member, O. Ogbuu, J. Liu, L. Zhang, and J. Michel, Journal of Lightwave Technology 31, 4029 (2013), arXiv:1304.1552.

${ }^{4}$ R. S. Jacobsen, K. N. Andersen, P. I. Borel, J. Fage-Pedersen, L. H. Frandsen, O. Hansen, M. Kristensen, A. V. Lavrinenko, G. Moulin, H. Ou, C. Peucheret, B. Zsigri, and A. Bjarklev, Nature 441, 199 (2006).

${ }^{5}$ B. Chmielak, M. Waldow, C. Matheisen, C. Ripperda, J. Bolten, T. Wahlbrink, M. Nagel, F. Merget, and H. Kurz, Optics express 19, 17212 (2011). 
${ }^{6}$ B. Chmielak, C. Matheisen, C. Ripperda, J. Bolten, T. Wahlbrink, M. Waldow, and H. Kurz, Optics Express 21, 25324 (2013)

${ }^{7}$ P. Damas, X. Le Roux, D. Le Bourdais, E. Cassan, D. MarrisMorini, N. Izard, T. Maroutian, P. Lecoeur, and L. Vivien, Optics Express 22, 22095 (2014).

${ }^{8}$ M. W. Puckett, J. S. T. Smalley, M. Abashin, A. Grieco, and Y. Fainman, Optics letters 39, 1693 (2014).

${ }^{9}$ M. Cazzanelli, F. Bianco, E. Borga, G. Pucker, M. Ghulinyan, E. Degoli, E. Luppi, V. Véniard, S. Ossicini, D. Modotto, S. Wabnitz, R. Pierobon, and L. Pavesi, Nature materials 11, 148 (2012).

${ }^{10}$ C. Schriever, F. Bianco, M. Cazzanelli, M. Ghulinyan, C. Eisenschmidt, J. de Boor, A. Schmid, J. Heitmann, L. Pavesi, and J. Schilling, Advanced Optical Materials 3, 129 (2015).

${ }^{11}$ S. S. Azadeh, F. Merget, M. P. Nezhad, and J. Witzens, Optics letters 40, 1877 (2015).
${ }^{12}$ R. Sharma, M. W. Puckett, H.-H. Lin, F. Vallini, and Y. Fainman, Applied Physics Letters 106, 241104 (2015).

${ }^{13}$ R. Sharma, M. W. Puckett, H.-H. Lin, A. Isichenko, F. Vallini, and Y. Fainman, Opt. Lett. 41, 1185 (2016).

${ }^{14}$ C. L. Manganelli, P. Pintus, and C. Bonati, Optics Express 23, 28649 (2015), arXiv:1507.06589.

${ }^{15}$ S. M. Sze and K. K. Ng., Physics of Semiconductor Devices, college edition ed. (Wilew-Interscience, 1976).

${ }^{16} \mathrm{R}$. Soref and B. Bennett, IEEE Journal of Quantum Electronics 23, 123 (1987).

${ }^{17}$ G. T. Reed, G. Mashanovich, F. Y. Gardes, and D. J. Thomson, Nature Photonics 4, 518 (2010).

${ }^{18}$ N. K. Hon, R. Soref, and B. Jalali, Journal of Applied Physics 110, 011301 (2011).

${ }^{19}$ P. Damas, D. Marris-Morini, E. Cassan, and L. Vivien, Physical Review B 93, 165208 (2016).

${ }^{20}$ M. Borghi, M. Mancinelli, F. Merget, J. Witzens, M. Bernard, M. Ghulinyan, G. Pucker, and L. Pavesi, Optics Letters 40, 5287 (2015). 